\title{
Plitidepsin: design, development, and potential place in therapy
}

\author{
This article was published in the following Dove Press journal: \\ Drug Design, Development and Therapy \\ 19 January 2017 \\ Number of times this article has been viewed
}

\author{
Sara Alonso-Álvarez' \\ Emilia Pardal ${ }^{2}$ \\ Diego Sánchez-Nieto ${ }^{3}$ \\ Miguel Navarro 4 \\ Maria Dolores Caballero' \\ Maria Victoria Mateos' \\ Alejandro Martín' \\ 'Hematology Department, IBSAL- \\ CIC-USAL, Hospital Universitario \\ de Salamanca, Salamanca, Spain; \\ ${ }^{2}$ Hematology Department, Hospital \\ Virgen del Puerto, Plasencia, Spain; \\ ${ }^{3}$ Pharmacy Department, Hospital \\ Universitario de Salamanca, Salamanca, \\ Spain; ${ }^{4}$ Oncology Department, \\ Hospital Universitario de Salamanca, \\ IBSAL, Salamanca, Spain
}

Correspondence: Alejandro Martín; Maria Victoria Mateos Department of Hematology, University Hospital of Salamanca, Paseo de San Vicente, 58-182, Salamanca, 37007, Spain Tel +34 $923291 \quad 100$ ext 55384 Fax +34923294624 Email amartingar@usal.es; mvmateos@usal.es

\begin{abstract}
Plitidepsin is a cyclic depsipeptide that was first isolated from a Mediterranean marine tunicate (Aplidium albicans) and, at present, is manufactured by total synthesis and commercialized as Aplidin $^{\circledR}$. Its antitumor activity, observed in preclinical in vitro and in vivo studies has prompted numerous clinical trials to be conducted over the last 17 years, alone or in combination with other anticancer agents. Single-agent plitidepsin has shown limited antitumor activity and a tolerable safety profile in several malignancies, such as noncutaneous peripheral T-cell lymphoma, melanoma, and multiple myeloma. In patients with relapsed or refractory multiple myeloma, plitidepsin activity seems to be enhanced after addition of dexamethasone while remaining well tolerated, and a Phase III trial comparing plitidepsin plus dexamethasone vs dexamethasone alone is underway. Additional studies are required to better define the role of plitidepsin in combination with other active agents in these indications. Results of plitidepsin activity in other hematological malignancies or solid tumors have been disappointing so far. Further studies analyzing its mechanisms of action and potential biomarkers will help select patients who may benefit most from this drug. In this review, we critically analyze the published studies on plitidepsin in hematological malignancies and solid tumors and discuss its current role and future perspectives in treating these malignancies. We also review its design, pharmaceutical data, and mechanism of action.
\end{abstract}

Keywords: aplidin, plitidepsin, lymphoma, myeloma, melanoma

\section{Design}

\section{Pharmaceutical data}

Aplidin $^{\circledR}$ (plitidepsin; PharmaMar, S.A., Madrid, Spain), the trade name for the active substance plitidepsin, is a synthetically produced anticancer agent. It is a cyclic depsipeptide that is structurally related to didemnins (Figure 1) and was originally isolated from a Mediterranean marine tunicate, Aplidium albicans. Its molecular formula is $\mathrm{C}_{57} \mathrm{H}_{87} \mathrm{~N}_{7} \mathrm{O}_{15}$ and it has a molecular weight of $1,110.34 .^{1-4}$

Plitidepsin was developed in the form of a lyophilized dosage form due to its substantial degradation under heat and light stress testing conditions when solubilized. The optimal reconstitution solution was found to be $15 / 15 / 70 \%(\mathrm{v} / \mathrm{v} / \mathrm{v})$ polyoxyl 35 castor oil/ethanol/water for injection. The reconstituted product and its dilutions with normal saline (up to $1: 100 \mathrm{v} / \mathrm{v}$ ) appeared to be stable for at least 24 hours after preparation. $^{5}$

The plitidepsin drug product is supplied as a powder to be made into a concentrated solution for infusion at a concentration of $2 \mathrm{mg} / \mathrm{vial}$. It is recommended that vials be reconstituted with $4 \mathrm{~mL}$ of the appropriate solution. The resulting solution must be clear and essentially free of visible particles. It may be colorless, but can turn slightly yellow. The reconstituted solution contains $0.5 \mathrm{mg} / \mathrm{mL}$ of plitidepsin (Table 1) and 


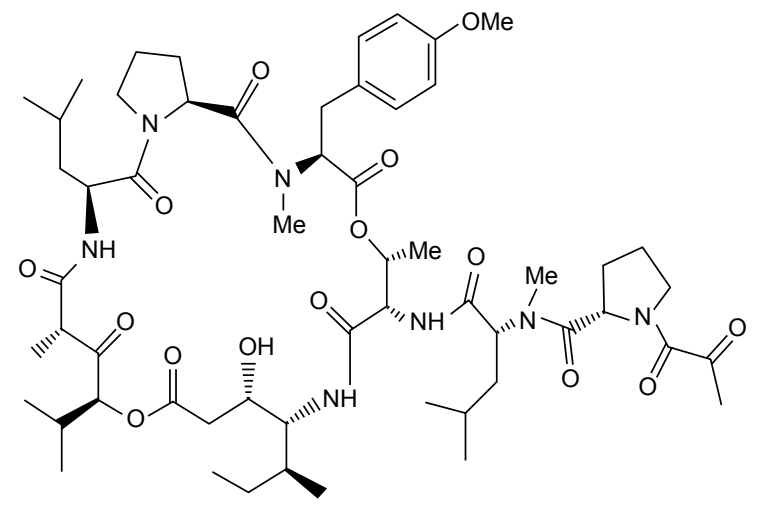

Figure I Plitidepsin chemical structure.

requires further dilution with $9 \mathrm{mg} / \mathrm{mL}$ sodium chloride $(0.9 \%)$ or $50 \mathrm{mg} / \mathrm{mL}$ glucose $(5 \%)$ infusion solution for administration as an intravenous infusion.

Plitidepsin and the reconstitution solution should be stored under the same conditions, between $2^{\circ} \mathrm{C}$ and $8^{\circ} \mathrm{C}$. Once reconstituted, the solution is physically and chemically stable for 28 hours up to $25^{\circ} \mathrm{C}$ under normal light conditions. However, it is recommended that it be diluted and used immediately. After dilution, the solution is stable for only 24 hours at temperatures of up to $25^{\circ} \mathrm{C}$ under normal light conditions.

\section{Mechanism of action}

The mechanism of action of plitidepsin IN various tumor cell types has been reviewed already and is represented in Figure 2. ${ }^{3}$ Plitidepsin induces dose-dependent cell-cycle arrest and an acute apoptotic process. These effects rely on the induction of early oxidative stress, the rapid activation of Rac1 GTPase, and the sustained activation of c-Jun $N$-terminal kinase (JNK) and p38 mitogen-activated protein kinases (p38/MAPK), which finally result in caspasedependent apoptosis. ${ }^{2,3} \mathrm{JNK}$ phosphorylation can be seen as early as 5-10 minutes after exposure to the compound. The activation of JNK and p38/MAPK is associated with an increase in the number of reactive oxygen species and a reduction in GSH. ${ }^{6}$

Table I Composition of reconstituted solution

\begin{tabular}{ll}
\hline Active substance & Amount per $\mathbf{~ m L}$ \\
\hline Plitidepsin & $0.5 \mathrm{mg}$ \\
Excipients & \\
D-Mannitol & $25.0 \mathrm{mg}$ \\
Polyoxyl 35 castor oil & $0.15 \mathrm{~mL}$ \\
Absolute ethanol & $0.15 \mathrm{~mL}$ \\
Water for injection & $0.70 \mathrm{~mL}$ \\
\hline
\end{tabular}

In addition, plitidepsin modifies the microenvironment that facilitates tumor growth. This was clearly observed in chronic lymphocytic leukemia (CLL) models. ${ }^{7}$ Plitidepsin not only directly affected the malignant B-CLL clone, but also had potent activity against monocytes and nurse-like cells (NLCs), a subset of cells differentiated from monocytes that favors leukemic cell progression. This effect is induced by triggering apoptosis, as evidenced by early exposure of phosphatidylserine in the outer leaflet of the plasma membrane, the activation of caspase-3, and the subsequent cleavage of PARP.

Antiangiogenic effects have been also reported for this drug. Broggini et $\mathrm{al}^{8}$ showed that plitidepsin blocks the secretion of the angiogenic factor vascular endothelial growth factor (VEGF) by the human leukemia cells MOLT-4, suggesting a possible effect on tumor angiogenesis. In another study, the same group showed that plitidepsin has antiangiogenic activity in vivo, in the chick embryo allantoic membrane assay, and inhibits endothelial cell functional responses to angiogenic stimuli in vitro. ${ }^{9}$ This effect might contribute to the antineoplastic activity of plitidepsin. ${ }^{7}$ In addition, a recent study have shown that the plitidepsin analogs PM01215 and PM02781 inhibit angiogenesis in vitro and in vivo. ${ }^{10}$

Recent studies have led researchers to hypothesize that the primary target of plitidepsin could be the eukaryotic Elongation Factor 1A2 (eEF1A2), ${ }^{11}$ which is overexpressed in tumors, including multiple myeloma (MM) and plasmacytoma, prostate, pancreas, and ovarian cancers, and that it exhibits oncogenic behavior by favoring tumor cell proliferation while inhibiting apoptosis. ${ }^{12}$ eEF1A2 is therefore an interesting target for cancer treatment and may also be a biomarker predicting drug sensitivity. The existence of an interaction between plitidepsin and eEF1A2 is suggested by several findings: ${ }^{11}$ 1) plitidepsin interacts with GTP-bound eEF1A2 with a $\mathrm{Kd}$ in the nanomolar range, compatible with the concentrations at which the compound shows its proapoptotic effect in tumor cells; 2) eEF1A2 is the only protein retrieved from a tumor cell lysate through a plitidepsin-binding fractionation process; 3) eEF1A2 is commonly depleted in plitidepsin-resistant cells, and its restitution to normal levels resensitizes them to the compound. These results suggest that eEF1A2 is the primary target of plitidepsin, which acts by mediating its antitumor activity.

\section{Preclinical studies}

In vitro studies showed that plitidepsin has cytotoxic activity against a broad range of cell lines showing $\mathrm{IC}_{50}$ values 


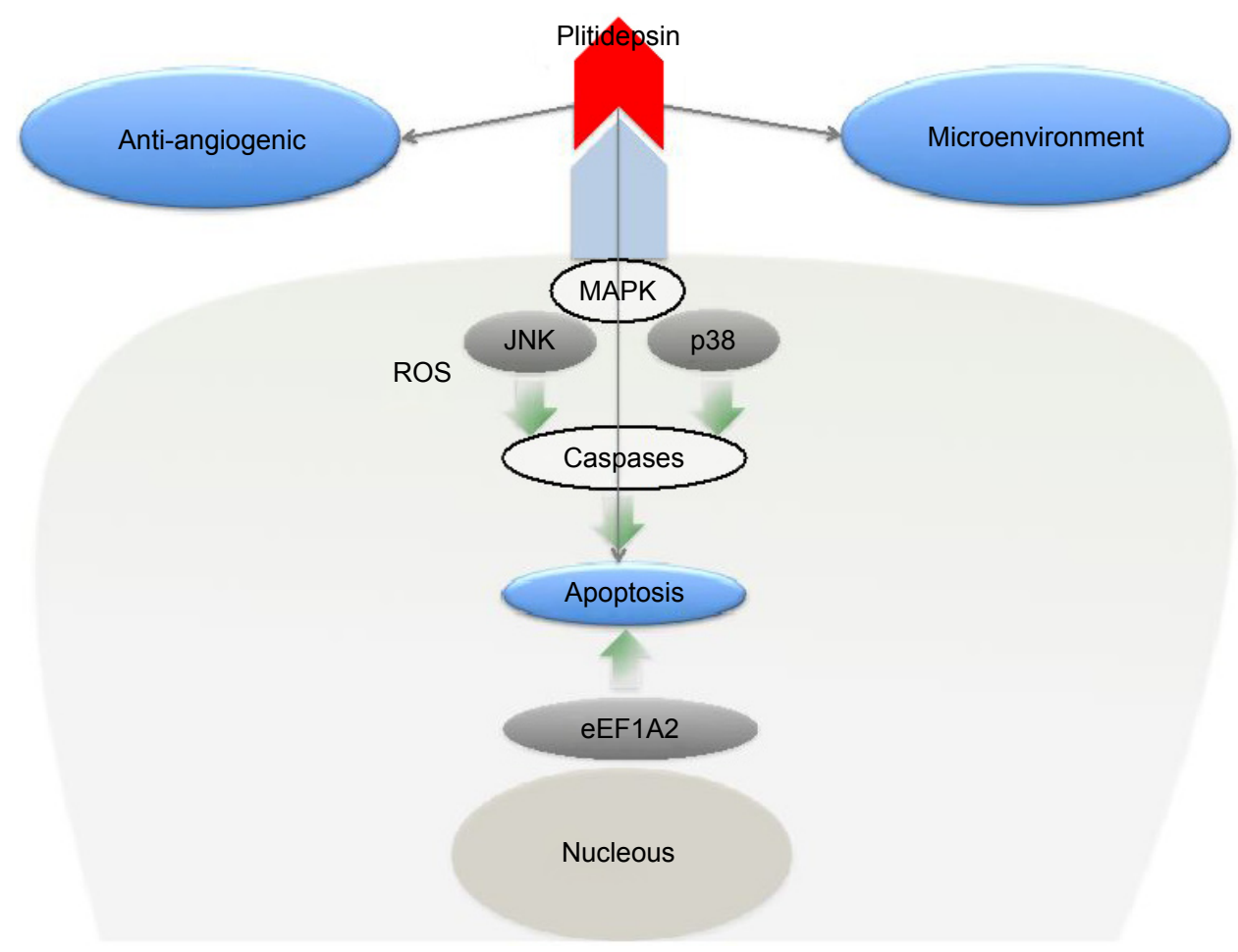

Figure 2 Plitidepsin mechanism of action.

of $\leq 1 \mathrm{nM}$. Its effects are particularly active against hematological malignancies such as MM, lymphoma and leukemia, and solid tumors including those of non-small-cell lung, pancreas, breast, melanoma, sarcoma, gastric, ovarian, bladder, and colon cancer. ${ }^{13-15}$ In addition, in vitro combination studies revealed a synergism of plitidepsin when combined with other agents like melphalan, dexamethasone, lenalidomide and bortezomib in MM cell lines. ${ }^{13}$

In vivo studies showed antitumor effect of plitidepsin in xenograft models of MM (as a single agent or in combination with dexamethasone), T-cell lymphoma, diffuse large B-cell lymphoma (DLBCL), Burkitt lymphoma (alone or in combination with rituximab), and pancreatic cancer (synergistic antitumor activity in combination with gemcitabine). ${ }^{16-19}$ The preclinical toxicological profile of plitidepsin is characteristic of a cytotoxic drug for which the liver, gastrointestinal tract, spleen, and bone marrow are the main target organs.

Presently, the hydrophobic drug plitidepsin is formulated using Cremophor ${ }^{\circledR}$, an adjuvant associated with unwanted hypersensitivity reactions..$^{20,21}$ In search of alternatives, two nanoparticle-based formulations of plitidepsin have been tested in a renal xenograft tumor model, ${ }^{22}$ showing equivalent anticancer activity, compared to the Cremophor ${ }^{\circledR}$ formulation, but improved biodistribution profiles, presenting novel tools for future plitidepsin-based therapies. Also, in colorectal cancer cell lines, the use of epidermal growth factor receptor (EGFR) targeted polymersomes greatly reduced plitidepsin cytotoxicity as well as the cellular uptake, indicating that the use of this targeted nanocarrier is a promising approach to tackle colorectal cancer disease and avoid the undesired effects of the usual treatment. ${ }^{23}$

\section{Pharmacokinetics and metabolism}

Several Phase I dose-escalation studies in subjects with advanced malignancies have described the pharmacokinetics of intravenous plitidepsin used as a single agent. Doses ranging from 0.13 to $8.00 \mathrm{mg} / \mathrm{m}^{2}$ were administered as a 1- or 24-hour infusion weekly, 3- or 24-hour infusion biweekly or, 1-hour infusion on 5 consecutive days every 3 weeks. In these studies, linear and time-independent pharmacokinetics in plasma were observed after several cycles of administration. ${ }^{24,25}$

After intravenous administration, plitidepsin was found to have a relatively long half-life, low clearance, and a high volume of distribution in plasma. In plasma and whole blood, mean half-lives ranged from 21 to 44 hours. Plitidepsin is widely distributed, with apparent volumes of distribution at steady state $(V$ ss $)$ ranging from 500 to 1,350 L. Approximately $97.3 \%$ of the human plasma protein was bound. ${ }^{26}$ 
Mean clearance values in plasma range from 45 to $49 \mathrm{~L} / \mathrm{h}$. Urinary excretion of the unchanged compound is a minor elimination route. ${ }^{25-27}$ Preliminary results of metabolism studies indicated that plitidepsin undergoes moderate microsomal-mediated metabolism in several animal species and humans. ${ }^{25}$

\section{Development Phase I trials}

Phase I clinical trials with plitidepsin were started in 1998. These studies, summarized in Table 2, were conducted according to classic Phase I study design standards. Fortnightly, weekly, or daily (days 1-5) schedules were used. Included patients were diagnosed with various solid tumors or non-Hodgkin lymphoma with relapsed or refractory disease. Plitidepsin was evaluated as a single agent in six trials (one of them in children), in which it consistently showed limited efficacy (Table 2). ${ }^{26-34}$ Overall, in 252 patients, two confirmed partial responses (PRs) and four unconfirmed PRs were found from all Phase I studies of plitidepsin. Clinical benefit, defined as stable disease (SD) lasting more than 3 months (time to progression [TTP] $>3$ months) was observed in 30 adult patients (range of clinical benefit, 3.0-9.7 months) and six children (range, 3.5-13.0 months). APL-A-005-02 was a multicenter, open-label, Phase I-II study to determine the maximum tolerated dose and recommended dose of plitidepsin as a single agent in 38 children with refractory or relapsed malignant tumors. Most $(n=31)$ discontinued treatment due to disease progression, but six children showed clinical benefit (Table 2). ${ }^{30}$

Plitidepsin in combination with other antineoplastic drugs has been evaluated in six Phase I studies ${ }^{31,32,35,36}$ (Table 2). Plitidepsin plus carboplatin (APL-A-006-05) resulted in an SD $>3$ months (range, 3.0-7.1 months) in six of 20 patients with advanced malignant solid tumors or lymphoma. ${ }^{31}$ Plitidepsin in combination with cytarabine has been tested in a Phase I/II trial in patients with relapsed/refractory leukemia, but the study was concluded early due to low recruitment. Plitidepsin and dacarbazine (Phase I stage of APL-B-016-05) resulted in one confirmed $\mathrm{PR}$, two unconfirmed PRs, and four SDs $>3$ months in 28 patients with unresectable advanced melanoma as frontline therapy. ${ }^{32}$ Bortezomib and dexamethasone have been added to plitidepsin in a Phase I/II trial (APL-A-012-13) in patients with relapsed and/or refractory MM. The recruitment has been completed $(\mathrm{N}=20)$ and the results are encouraging, with $55 \%$ overall response rate (ORR) in a heavily pretreated population in which $88 \%$ were bortezomib exposed and $61 \%$ were refractory to the last line of therapy. In addition, toxicity profile was manageable, so the Phase II is ongoing. ${ }^{36}$

\section{Phase II trials}

Results of plitidepsin Phase II trials are summarized in Table 3. Two different schedules were used, based on the findings from previous Phase I studies. The most common scheme, which was used in 10 of the existing Phase II trials, was the fortnightly scheme, consisting of plitidepsin $5 \mathrm{mg} / \mathrm{m}^{2}$ administered as a 3-hour intravenous infusion on days 1 and 15, every 4 weeks. A 24-hour infusion was used in the first Phase II study performed (APL-B-001-01), ${ }^{34}$ with a higher dose (up to $7 \mathrm{mg}$ / $\mathrm{m}^{2}$ ) of plitidepsin as a single agent or in combination with L-carnitine. The second scheme was the weekly schedule, used in four Phase II studies, consisting of plitidepsin $3.2 \mathrm{mg} / \mathrm{m}^{2}$ administered as a 1-hour intravenous infusion on days 1,8 , and 15 , every 4 weeks. One study (APL-B-016-05) ${ }^{32}$ used lower doses of plitidepsin $\left(2.4 \mathrm{mg} / \mathrm{m}^{2}\right)$ and was given in combination with dacarbazine at a dose of $800 \mathrm{mg} / \mathrm{m}^{2}$.

With respect to hematological malignancies, a Phase II trial (APL-B-020-10) of 11 patients with primary or secondary myelofibrosis was concluded early due to lack of efficacy. ${ }^{37}$ Antitumor activity seems to be greater in lymphoid neoplasms. Fifty one patients with relapsed or refractory MM were included in a Phase II trial (APL-B-014-03) with plitidepsin at $5 \mathrm{mg} / \mathrm{m}^{2}$ as a 3-hour intravenous (IV) infusion every 2 weeks. The protocol was amended to allow patients with a suboptimal response to single-agent plitidepsin to add $20 \mathrm{mg} / \mathrm{d}$ of oral dexamethasone on days $1-4$, every 2 weeks. From 47 patients evaluable for efficacy, the ORR (complete remission plus PR plus minimal response) was $13 \%$ with plitidepsin alone, and $22 \%$ in the cohort of patients with the addition of dexamethasone ( $n=19,18$ evaluable). Plitidepsin alone and with dexamethasone were feasible and well tolerated in this heavily pretreated population. ${ }^{36}$ The activity observed with plitidepsin after dexamethasone addition merits further study.

In another Phase II trial (APL-B-013-02), 67 patients with relapsed or refractory aggressive non-Hodgkin lymphoma received plitidepsin according to the weekly scheme. Patients were divided into two cohorts: those with noncutaneous peripheral T-cell lymphoma (PTCL) $(n=34)$ who received a median of two cycles (range, $1-8$ cycles) per patient, and those with other lymphomas $(n=33)$ who received a median of 1.5 cycles (range, 1-4 cycles). Efficacy was evaluated using the International Working Group criteria (1999). Of the 29 evaluable patients with noncutaneous PTCL, six had a response (ORR 20.7\%), including two complete remissions (both angioimmunoblastic T-cell lymphomas pretreated with 


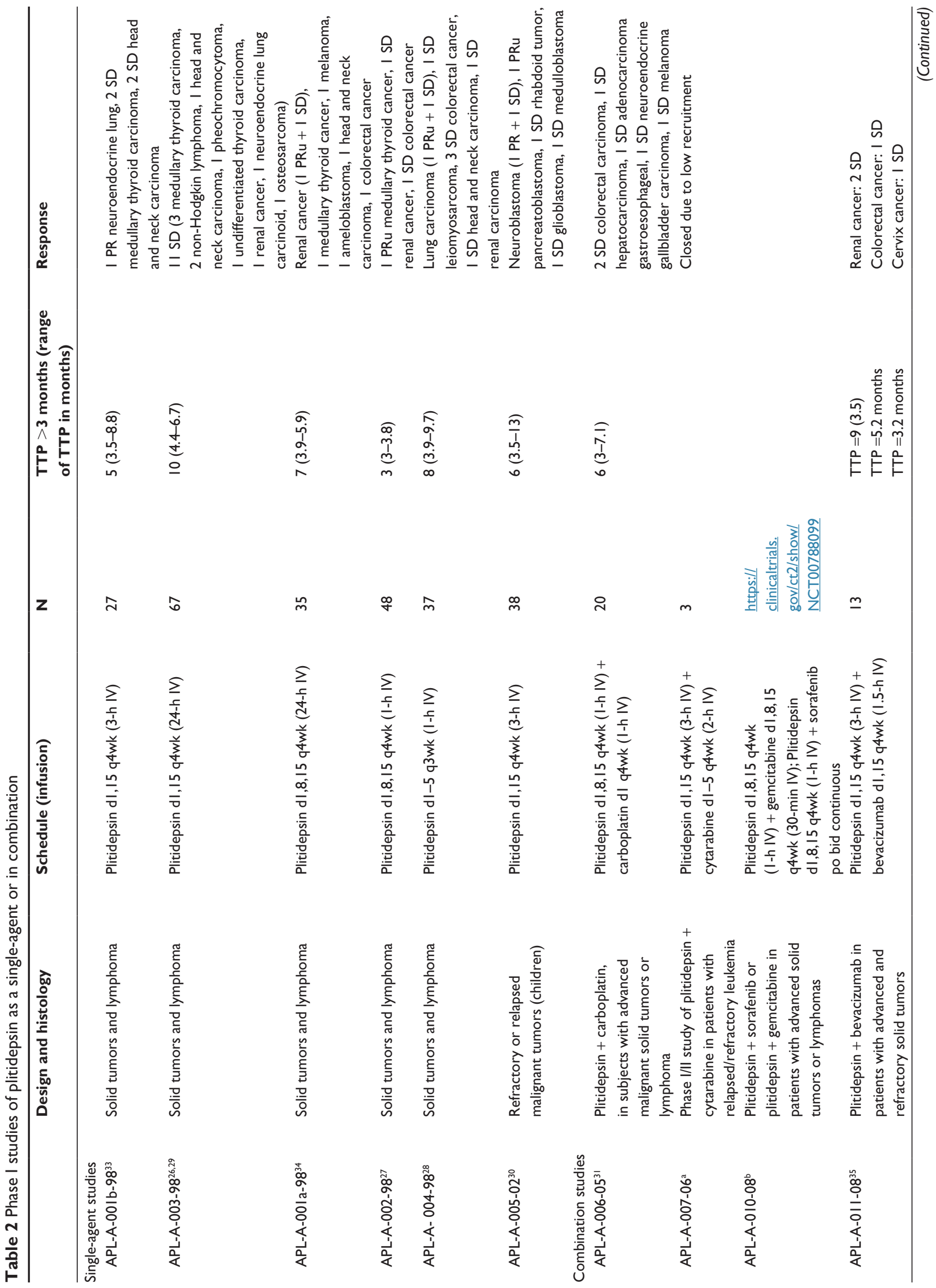




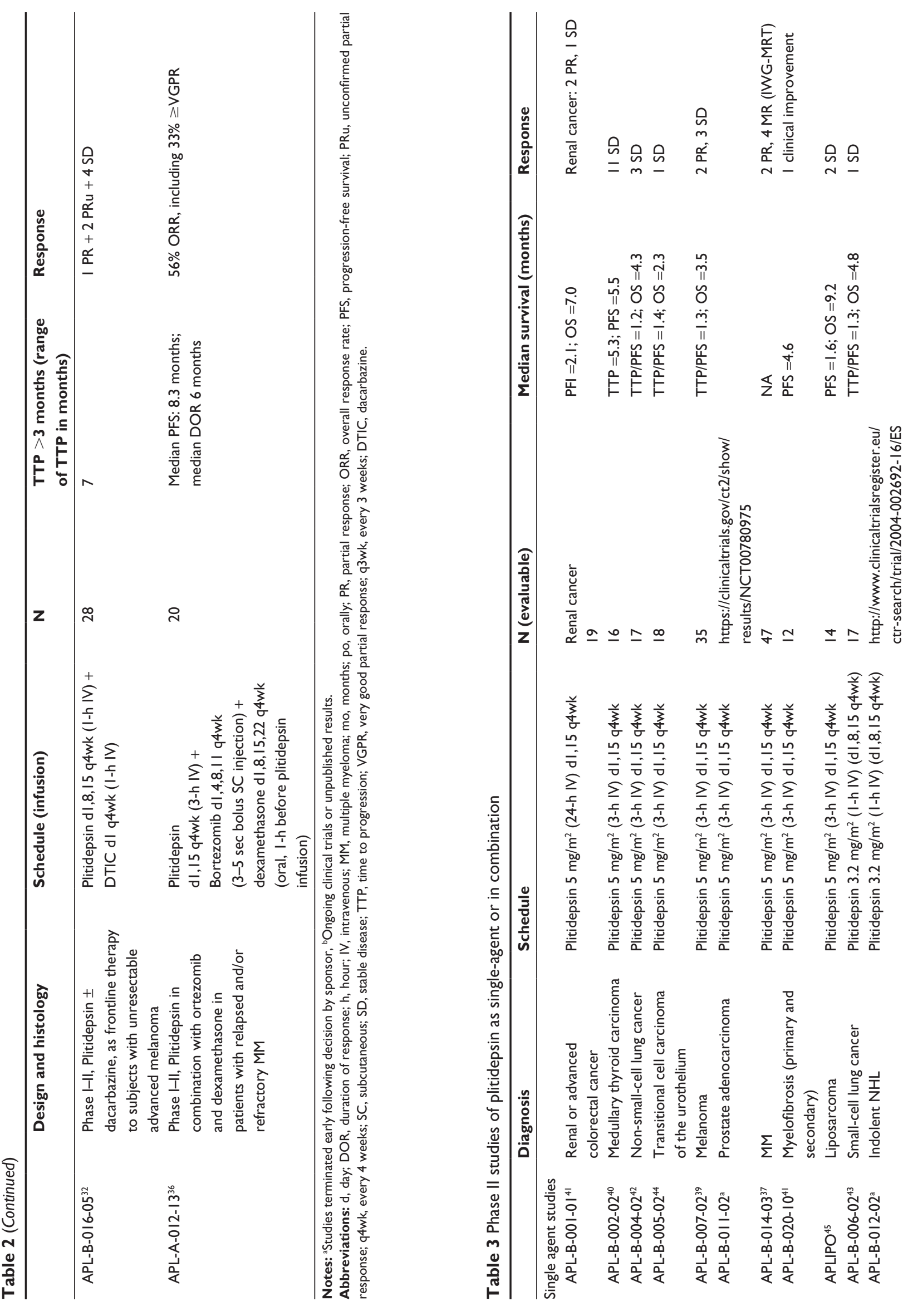




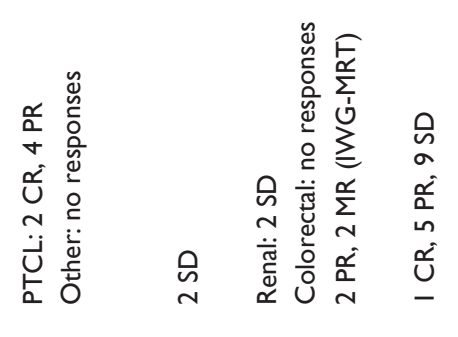

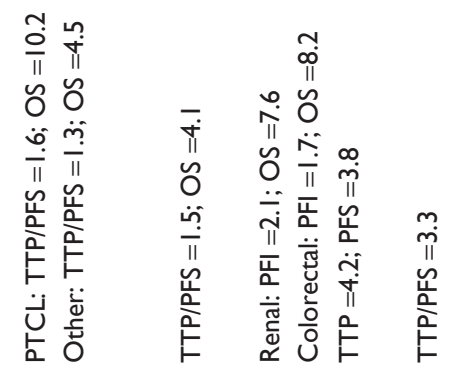

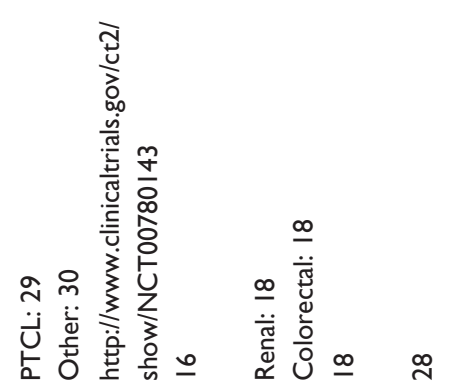

\begin{tabular}{|c|c|c|c|}
\hline 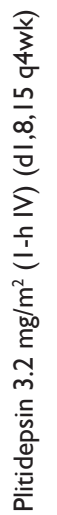 & 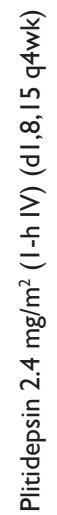 & 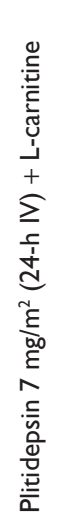 & 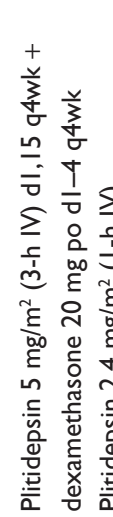 \\
\hline
\end{tabular}

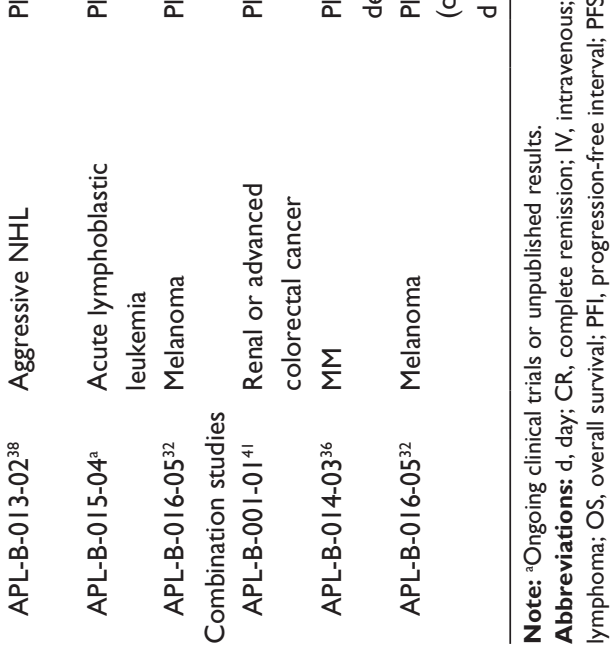

stem-cell transplantation) and four PRs. The median duration of response (DOR) and TTP were 2.2 and 1.6 months, respectively. No responses occurred in the 30 evaluable patients with other lymphomas (including 27 B-cell lymphomas). ${ }^{39}$

With respect to solid tumors, plitidepsin has shown a low level of antitumor activity in patients with malignant melanoma. In a Phase II study, 37 patients with advanced malignant melanoma who relapsed or progressed after one line of systemic therapy received plitidepsin according to the fortnightly scheme. The objective response rate (primary efficacy end point) was evaluated according to the Response Evaluation Criteria In Solid Tumors. Of the 35 evaluable patients, two dacarbazine-resistant patients (5.7\%) with metastatic cutaneous melanoma achieved PR. Five other patients (14.3\%) reported SD (median SD duration, 3.5 months; range, 2.2-15.8 months). Therefore, the rate of tumor growth control was $20.0 \%$. With a median follow-up of 11.0 months, the median progression-free survival (PFS) was 1.3 months and the median overall survival (OS) was 3.5 months. ${ }^{40}$ These results suggest that further evaluation of plitidepsin in combination schedules is warranted. A Phase I/II trial compared plitidepsin alone or combined with dacarbazine (DTIC) as frontline therapy for advanced melanoma. The ORR with plitidepsin/DTIC was $21.4 \%$; all responders had normal serum lactate dehydrogenase (LDH) levels and performance status $\leq 1$ at baseline. Median PFS with plitidepsin/DTIC was 3.3 months in all patients and 4.3 months in those with baseline normal LDH. No responses occurred with single-agent plitidepsin - median PFS was 1.5 months. Both regimens were well tolerated. Further evaluation of plitidepsin $2.4 \mathrm{mg} / \mathrm{m}^{2}$ fortnightly and DTIC $800 \mathrm{mg} / \mathrm{m}^{2} \mathrm{q} 4 \mathrm{wk}$ was recommended by the authors. ${ }^{32}$

Plitidepsin as a single agent has shown very limited or no antitumor activity in other solid tumors. Results from other malignancies comprised: two PRs and one SD among 19 patients with unresectable advanced renal cell carcinoma, with a 2.1-month progression-free interval (PFI) (APL-B-001-01); ${ }^{41} 11$ SDs out of 16 patients with unresectable advanced medullary thyroid carcinoma, with a clinical benefit noted in only one patient (APL-B-002-02);2 three SDs in 17 patients with locally advanced or metastatic non-smallcell lung cancer, with a TTP of 1.2 months (APL-B-004-02); ${ }^{43}$ no responses in another trial of 19 patients with small-cell lung cancer pretreated with one line (APL-B-006-02); ${ }^{44}$ one SD as second-line therapy for 18 patients with advanced or metastatic transitional cell carcinoma of the urothelium (APL-B-005-02), ${ }^{45}$ two SDs among 22 assessable patients with advanced dedifferentiated liposarcomas, with a median 
PFS of 1.6 months (APIPLO study). ${ }^{46}$ Coadministration of L-carnitine did not increase treatment efficacy and did not prevent the muscular toxicity or CPK-elevation associated with plitidepsin in patients with unresectable advanced renal cell carcinoma. ${ }^{41}$

\section{Phase III trials}

According to the encouraging preliminary results with plitidepsin plus dexamethasone in MM, a multicenter, open-label, randomized, Phase III clinical trial (ADMYRE), which started in June 2010 and finished recruitment in June 2015, has compared the efficacy and safety of plitidepsin combined with dexamethasone vs dexamethasone alone in patients with relapsed/refractory MM previously treated with between three and six therapeutic regimens. Efficacy data are currently being analyzed. A total of 255 patients received either plitidepsin $5 \mathrm{mg} / \mathrm{m}^{2} \mathrm{IV}$ as a 3-hour infusion on days 1 and $15 \mathrm{q} 4 \mathrm{wk}$ plus dexamethasone $40 \mathrm{mg}$ orally on days 1, 8, 15 and 22 q4wk (Arm A), or dexamethasone alone at the same dose and schedule (Arm B). A press release has recently reported the significant benefit of plitidepsin plus dexamethasone over dexamethasone alone (a 35\% lower risk of disease progression or death), and we await the final results.

\section{Toxicities in Phase I studies}

The primary dose-limiting toxicities (DLTs) found in the dose-finding Phase I studies with single-agent plitidepsin were musculoskeletal adverse events (AEs). ${ }^{26-30,36,41}$ The most common were myalgia, muscle weakness, and increase in serum CPK (noncardiac fraction) levels. Myalgia usually started proximally (shoulders, neck or thighs) at about 3 weeks after treatment onset, whereas muscle weakness and increases in serum CPK levels usually started about 2 weeks later. Myalgia and muscle weakness both generally resolved within 1-3 weeks. Increases in asymptomatic CPK may occur, as well as myalgia or muscle cramps, with no increase in CPK levels. No deaths or life-threatening consequences occurred due to these events. No rhabdomyolysis, increase of blood myoglobin levels, or myoglobinuria were reported from the administration of plitidepsin either as a single-agent or in combinations. Increased blood CPK levels were the only serious laboratory abnormality related to muscular disorders reported in single-agent Phase I studies.

The other relevant DLT in adult patients was hepatic toxicity, which manifested most frequently as transient and reversible increases in transaminase (particularly ALT). ${ }^{32.36}$
In Phase II studies, these abnormalities were asymptomatic, reversible, and noncumulative.

Hematological abnormalities were not DLTs in any of the schedules used in Phase I studies. As a single-agent, plitidepsin did not induce clinically significant bone marrow toxicity, stomatitis, or alopecia within the dose range explored. It is of note that neither grade $3 / 4$ neutropenia nor thrombocytopenia were reported in any Phase I trial with plitidepsin as a single agent.

Grade 1/2 vomiting occurred in about half of the patients treated at the recommended dose. The use of prophylactic treatment for emesis resulted in a low incidence of grade $3 / 4$ vomiting in Phase II studies (2\%). General symptoms (eg, fatigue) were also reported during treatment with plitidepsin. Mild infusion site reactions were found, particularly (although not exclusively) when plitidepsin was administered through a peripheral line.

The most common DLTs found with the plitidepsin combination schedules ${ }^{32.36}$ were transaminase increases. DLTs consisting of hematological abnormalities were uncommon.

In the single-agent Phase I trial in pediatric patients (APLA-005-02), ${ }^{30}$ the safety profile was not strikingly different from that in the adult Phase I program, although a slightly higher incidence of grade 3/4 hypersensitivity reactions was observed despite mandatory prophylactic medication.

\section{Safety in completed Phase II trials}

\section{Treatment exposure}

Four hundred and forty patients were treated in Phase II trials (288 patients diagnosed with solid tumors and 152 with hematological malignancies) with 1,646 cycles administered. Two hundred and seventy six patients (1,283 cycles) were treated with the fortnightly schedule, and 164 patients (363 cycles) with the weekly schedule..$^{32,37-45}$

\section{Muscular adverse events}

Muscular events were described in about $50 \%$ of patients treated in Phase II studies, sharing similar characteristics to those seen in Phase I studies. Globally, muscle toxicity induced by plitidepsin is generally mild to moderate, manageable, and reversible upon treatment discontinuation or dose reduction, without requiring additional therapeutic measures. Myalgia occurred in about $40 \%$ of patients with solid tumors and $17 \%$ of those with hematological malignancies. Muscular weakness was the second most common muscular AE. Shorter infusion times may help reduce the incidence of 
some types of drug-related events, including CPK elevations, gastrointestinal (anorexia, nausea, and vomiting), constitutional (fatigue), and injection-site reactions.

\section{Hematological abnormalities}

Most hematological abnormalities were mild to moderate. As expected, grade $3 / 4$ toxicities occurred more often in patients with hematological malignancies than in patients with solid tumors. Most importantly, no grade 3/4 neutropenia occurred in solid tumor patients, in contrast to $16 \%$ of patients with hematological malignancies (fortnightly schedule). Bone marrow toxicity has been more common in studies evaluating the 3-hour and 1-hour infusion schedules, due to the higher proportion of patients with hematological malignancies in these studies.

\section{Biochemical abnormalities}

Most biochemical abnormalities were mild or moderate. Grade 3/4 abnormalities were more frequent in patients with hematological malignancies than in those with solid tumors, including increases in ALT, AST, CPK, and creatinine. In contrast, increases in amylase and bilirubin were more common in patients with solid tumors.

\section{Metabolic abnormalities}

No substantial differences were found between schedules, or between patients with solid tumors and patients with hematological malignancies. Grade 1/2 hyperglycemia and hypoalbuminemia were common in association either with premedication (dexamethasone) or with the underlying advanced neoplastic disease, respectively. As expected, hypercalcemia was common in MM patients and was related to the underlying malignancy.

\section{Gastrointestinal adverse events}

Nausea, vomiting, and diarrhea were the most frequent gastrointestinal AEs. Most episodes were mild or moderate and reached grade $3 / 4$ in fewer than $2 \%$ of patients on the fortnightly schedule, but were more frequent among patients with solid tumors and those on the weekly schedule. Plitidepsin is defined as a treatment with high (non-cisplatin) emetogenic risk and appropriate primary prophylaxis is indicated.

\section{Constitutional symptoms}

Fatigue has been the most frequently reported constitutional adverse event in Phase II studies (41\%). It is more common $(49 \%)$ in patients with solid tumors than in those with hematological malignancies (21\%-38\%). Grade 3-4 fatigue was found less often $(1 \%-18 \%)$. Noninfectious pyrexia has been documented in $7 \%-11 \%$ of patients.

\section{Cardiac adverse events}

The most frequent type of AE was rhythm alterations, while the other events were relatively infrequent. No lifethreatening ventricular arrhythmias occurred. No fatal outcome was reported as a consequence of these cardiac AEs in Phase II trials. ${ }^{44,47}$

\section{Hypersensitivity reactions}

Drug-related hypersensitivity reactions were found in $10.7 \%$ of patients treated with plitidepsin in Phase I and II trials. The incidence of hypersensitivity reactions was similar between the different tumor types. Grade $3 / 4$ reactions occurred in $2.7 \%$, with or without previous prophylaxis. Due to these reactions, all subsequent Phase II trials since February 2004 have included premedication with both anti-H1 and anti-H2 type antihistamines and glucocorticoids. Despite this prophylaxis, the rate of occurrence of serious reactions in all patients has remained unchanged overall, and only grade 1 reactions have decreased. ${ }^{32,37-45}$ In a phase II study ${ }^{37}$ using plitidepsin and dacarbazine as first-line therapy for advanced melanoma, hypersensitivity reactions were increased when using the combination therapy compared to plitidepsin as single agent. Globally, serious reactions tend to occur slightly more frequently in younger patients, females, and possibly those on weekly schedules in subsequent exposures (these reactions were rarely seen in the first infusion). These reactions are rapidly reversible and, to date, none has had a fatal outcome.

\section{Other adverse events}

The incidence of other AEs, such as neurological or respiratory AEs, was low or very low.

\section{Deaths}

Almost all deaths that occurred during treatment or follow-up were due to progression of the underlying malignancy and were unrelated to plitidepsin. In Phase I studies only one death was drug related. No deaths due to toxicity have been reported in children. ${ }^{30}$

\section{Potential place in therapy}

Plitidepsin has shown mild-to-moderate antitumor activity, mainly in patients with MM, PTCL, and melanoma. Adverse 
events are manageable and predictable. The mild hematological toxicity associated with plitidepsin makes this drug an attractive option for combination with chemotherapy schedules. As previously mentioned, recent studies suggest that eEF1A $2^{11}$ is the primary target of plitidepsin mediating its antitumor activity and might be a biomarker predicting drug sensitivity, although further studies are needed to confirm this.

\section{Multiple myeloma}

Plitidepsin has shown limited, although reproducible, singleagent activity in relapsed/refractory MM, which seems to be enhanced after dexamethasone addition while remaining well tolerated. The results of the Phase I trial combining plitidepsin with bortezomib and dexamethasone support the continuous development of additional studies to better define the role of plitidepsin in combination with other active agents in this indication. After the results of the Phase II trial (NCT00229203, APL-B-014-03) ${ }^{36}$ evaluating plitidepsin alone or in combination with dexamethasone, a new registry Phase III, multicenter, open-label, randomized trial (NCT 01102426, ADMYRE, APL-C-001-09) ${ }^{48}$ was started in June 2010 with recruitment being completed in June 2015. This trial evaluated the efficacy and safety of plitidepsin combined with dexamethasone vs dexamethasone alone in 255 patients with relapsed/refractory MM previously treated with between three and six therapeutic regimens (bortezomib and lenalidomide-thalidomide included). Efficacy data from ADMYRE ${ }^{46}$ will support valuable information regarding which patients could benefit from this drug. A press release has recently reported that plitidepsin plus dexamethasone had a significantly 35\% lower risk of disease progression or death compared with dexamethasone. Based on these results, plitidepsin will be put forward for approval for use in Europe in this combination for the treatment of patients with MM.

\section{Lymphoma}

Single-agent plitidepsin has shown antitumor activity and a tolerable safety profile in patients with relapsed/refractory noncutaneous PTCL in a Phase II trial (APL-B-013-02). ${ }^{39}$ These results support the conduction of further clinical trials to evaluate plitidepsin-containing combined therapies in this disease setting. The absence of antitumor activity found in aggressive B-cell lymphomas ${ }^{39}$ precludes further studies of plitidepsin in this indication. A Phase II study of plitidepsin as a single-agent in indolent lymphomas is ongoing and the results are awaited.

\section{Solid tumors}

Plitidepsin is a potentially effective and safe drug for the treatment of different solid tumors in monotherapy or in combination with other drugs. In Phase II trials, plitidepsin has shown a low level of antitumor activity in patients with malignant melanoma. ${ }^{32,40}$ In this setting, plitidepsin could become a useful drug, although further trials are needed to better define its role in combination with other active agents such as immunotherapy or targeted therapies. Very limited or no antitumor activity of plitidepsin as a single-agent in other solid tumors has been noted to date. The absence of antitumor activity in small-cell lung cancer, ${ }^{43,44}$ transitional cell carcinoma of the urothelium, ${ }^{45}$ medullary thyroid carcinoma, ${ }^{29}$ and liposarcoma ${ }^{46}$ precludes further studies of plitidepsin for these indications, at least in the schedules used in the trials described.

\section{Conclusion}

Plitidepsin is a cyclic depsipeptide that was first isolated from a Mediterranean marine tunicate ${ }^{1}$ (Aplidium albicans) and, at present, is manufactured by total synthesis and commercialized as plitidepsin. Its antitumor activity, observed in preclinical in vitro and in vivo studies, has prompted numerous clinical trials during the last 17 years involving plitidepsin alone or in combination with other anticancer agents. It has a favorable and predictable toxicity profile and has limited although reproducible single-agent activity in relapsed/refractory MM, which seems to be enhanced after dexamethasone addition, while remaining well tolerated. A Phase III trial comparing plitidepsin plus dexamethasone vs dexamethasone alone is underway, and additional studies are awaited that better define its role in combination with other active agents in this indication. Single-agent plitidepsin shows moderate antitumor activity and a tolerable safety profile in patients with relapsed/refractory noncutaneous PTCL and in patients with melanoma in Phase II trials. Further clinical trials to evaluate combined therapies containing plitidepsin in these settings are warranted. Results of the use of plitidepsin in the treatment of other hematologic malignancies or solid tumors have been disappointing. Further studies analyzing its mechanisms of action and potential biomarkers will help identify those patients who might benefit most from this drug.

\section{Acknowledgment}

Maria Dolores Caballero, Maria Victoria Mateos, and Alejandro Martín should be conisidered as senior authors.

\section{Disclosure}

The authors report no conflicts of interest in this work. 


\section{References}

1. Rinehart KL. Antitumor compounds from tunicates. Med Res Rev. 2000;20:1-27.

2. Garcia-Fernandez LF, Losada A, Alcaide V, et al. Aplidin induces the mitochondrial apoptotic pathway via oxidative stress-mediated JNK and p38 activation and protein kinase C delta. Oncogene. 2002;21: 7533-7544.

3. Munoz-Alonso MJ, Gonzalez-Santiago L, Martinez T, et al. The mechanism of action of plitidepsin. Curr Opin Investig Drugs. 2009;10:536-542.

4. Urdiales JL, Morata P, Nunez De Castro I. Antiproliferative effect of dehydrodidemnin B (DDB), a depsipeptide isolated from Mediterranean tunicates. Cancer Lett. 1996;102:31-37.

5. Nuijen B, Bouma M, Henrar RE, et al. Pharmaceutical development of a parenteral lyophilized formulation of the novel antitumor agent aplidine. PDA J Pharm Sci Technol. 2000;54:193-208.

6. Gonzalez-Santiago L, Suarez Y, Zarich N, et al. Aplidin induces JNK-dependent apoptosis in human breast cancer cells via alteration of glutathione homeostasis, Rac1 GTPase activation, and MKP-1 phosphatase downregulation. Cell Death Differ. 2006;13: 1968-1981.

7. Morande PE, Zanetti SR, Borge M, et al. The cytotoxic activity of Aplidin in chronic lymphocytic leukemia (CLL) is mediated by a direct effect on leukemic cells and an indirect effect on monocyte-derived cells. Invest New Drugs. 2012;30:1830-1840.

8. Broggini M, Marchini SV, Galliera E, et al. Aplidine, a new anticancer agent of marine origin, inhibits vascular endothelial growth factor (VEGF) secretion and blocks VEGF-VEGFR-1 (flt-1) autocrine loop in human leukemia cells MOLT-4. Leukemia. 2003;17(1):52-59.

9. Taraboletti G, Poli M, Dossi R, et al. Antiangiogenic activity of aplidine, a new agent of marine origin. Br J Cancer. 2004;14;90(12): $2418-2424$.

10. Borjan B, Steiner N, Karbon S, et al. The Aplidin analogs PM01215 and PM02781 inhibit angiogenesis in vitro and in vivo. BMC Cancer. 2015; $15: 738$.

11. Losada A, Martínez-Leal JF, Gago F, et al. Role of the eukaryotic elongation factor eEF1A in the mechanism of action of Aplidin. Abstract 5467. Presented at: American Association for Cancer Research Annual Meeting; 2014; San Diego, CA.

12. Losada A, Lopez-Oliva JM, Sanchez-Puelles JM, Garcia-Fernandez LF. Establishment and characterisation of a human carcinoma cell line with acquired resistance to Aplidin. Br J Cancer. 2004;91:1405-1413.

13. Depenbrock H, Peter R, Faircloth GT, Manzanares I, Jimeno J, Hanauske AR. In vitro activity of aplidine, a new marine-derived anticancer compound, on freshly explanted clonogenic human tumour cells and haematopoietic precursor cells. Br J Cancer. 1998;78:739-744.

14. Faircloth G, Rinehart K, Nunez de Castro I, Jimeno J. Dehydrodidemnin B, a new marine derived antitumour agent with activity against experimental tumour models. Ann Oncol. 1996;7:9.

15. Faircloth G, Hnauske A, Depenbroch H, et al. Preclinical characterization of Aplidine (APD), a new marine anticancer depsipeptide (MADEP). Proc AACR. 1997;38:103-110.

16. Caers J, Menu E, De Raeve H, et al. Antitumour and antiangiogenic effects of Aplidin in the 5TMM syngeneic models of multiple myeloma. Br J Cancer. 2008;98:1966-1974.

17. Mitsiades CS, Ocio EM, Pandiella A, et al. Aplidin, a marine organismderived compound with potent antimyeloma activity in vitro and in vivo. Cancer Res. 2008;68:5216-5225.

18. Barboza NM, Medina DJ, Budak-Alpdogan T, et al. Plitidepsin (Aplidin) is a potent inhibitor of diffuse large cell and Burkitt lymphoma and is synergistic with rituximab. Cancer Biol Ther. 2012;13: 114-122.

19. Mishra P, Longo-Sorbello G, Mishra P, et al. Synergistic action of plitidepsin and gemcitabine against pancreatic cancer in vitro and in vivo. Abstract no. 4501. Presented at: Proceedings of 100th American Association for Cancer Research Meeting; 2009; Denver, CO.

20. Weiss RB, Donehower RC, Wiernik PH, et al. Hypersensitivity reactions from taxol. J Clin Oncol. 1990;8:1263-1268.
21. Gelderblom H, Verweij J, Nooter K, Sparreboom A. Cremophor EL: the drawbacks and advantages of vehicle selection for drug formulation. Eur J Cancer. 2001;37:1590-1598.

22. Goñi-de-Cerio F, Thevenot J, Oliveira H, et al. Cellular Uptake and Cytotoxic Effect of Epidermal Growth Factor Receptor Targeted and Plitidepsin Loaded Co-Polymeric Polymersomes on Colorectal Cancer Cell Lines. J Biomed Nanotechnol. 2015;11(11):2034-2049.

23. Oliveira H, Thevenot J, Garanger E, et al. Nano-encapsulation of plitidepsin: in vivo pharmacokinetics, biodistribution, and efficacy in a renal xenograft tumor model. Pharm Res. 2014;31(4):983-991.

24. Brandon EF, van Ooijen RD, Sparidans RW, et al. Structure elucidation of aplidine metabolites formed in vitro by human liver microsomes using triple quadrupole mass spectrometry. J Mass Spectrom. 2005; 40(6):821-831.

25. Faivre S, Chieze S, Delbaldo C, et al. Phase I and pharmacokinetic study of aplidine, a new marine cyclodepsipeptide in patients with advanced malignancies. J Clin Oncol. 2005;23:7871-7880.

26. Nalda-Molina R, Valenzuela B, Ramon-Lopez A, et al. Population pharmacokinetics meta-analysis of plitidepsin (Aplidin) in cancer subjects. Cancer Chemother Pharmacol. 2009;64:97-108.

27. Izquierdo MA, Bowman A, Garcia M, et al. Phase I clinical and pharmacokinetic study of plitidepsin as a 1-hour weekly intravenous infusion in patients with advanced solid tumors. Clin Cancer Res. 2008; 14:3105-3112.

28. Maroun JA, Belanger K, Seymour L, et al. Phase I study of Aplidine in a dailyx 5 one-hour infusion every 3 weeks in patients with solid tumors refractory to standard therapy. A National Cancer Institute of Canada Clinical Trials Group study: NCIC CTG IND 115. Ann Oncol. 2006;17: 1371-1378.

29. Le Tourneau C, Faivre S, Ciruelos E, et al. Reports of clinical benefit of plitidepsin (Aplidine), a new marine-derived anticancer agent, in patients with advanced medullary thyroid carcinoma. Am J Clin Oncol. 2010; 33:132-136

30. Geoerger B, Estlin EJ, Aerts I, et al. A Phase I and pharmacokinetic study of plitidepsin in children with advanced solid tumours: an Innovative Therapies for Children with Cancer (ITCC) study. Eur J Cancer. 2012; 48:289-296.

31. Salazar R, Plummer R, Oaknin A, et al. Phase I study of weekly plitidepsin as 1-hour infusion combined with carboplatin in patients with advanced solid tumors or lymphomas. Invest New Drugs. 2011;29:1406-1413.

32. Plummer R, Lorigan P, Brown E, et al. Phase I-II study of plitidepsin and dacarbazine as first-line therapy for advanced melanoma. $\mathrm{Br} J$ Cancer. 2013;109:1451-1459.

33. Ciruelos EM, Twelves C, Dominguez MJ, et al. Phase I clinical and pharmacokinetic study of the marine compound Aplidin (APL) administered as a 3 hour infusion every 2 weeks. Proc Am Soc Clin Oncol. 2002; 21: abst. 422.

34. Anthoney A, Paz-Ares L, Twelves C, et al. Phase I and pharmacokinetic (PK) study of Aplidin (APL) using a 24-hour weekly schedule. Proc Am Soc Clin Oncol. 2000;19: abst. 734.

35. Gomez-Roca C, Besse-Hammer T, Szyldergemajn S, et al. Phase IB study of plitidepsin (APL) with bevacizumab (BEV) in refractory solid tumor patients (pts). Eur J Cancer. 2010;8: abst. 432.

36. Ocio EM, Mateos MV, Prósper F, et al. Phase I study of plitidepsin in combination with bortezomib and dexamethasone in patients with relapsed and/or refractory multiple myeloma. J Clin Oncol. 2016; 34(suppl): abst. 8006.

37. Pardanani A, Tefferi A, Guglielmelli P, et al. Evaluation of plitidepsin in patients with primary myelofibrosis and post polycythemia vera/ essential thrombocythemia myelofibrosis: results of preclinical studies and a Phase II clinical trial. Blood Cancer J. 2015;5:e286.

38. Mateos MV, Cibeira MT, Richardson PG, et al. Phase II clinical and pharmacokinetic study of plitidepsin 3-hour infusion every two weeks alone or with dexamethasone in relapsed and refractory multiple myeloma. Clin Cancer Res. 2010;16:3260-3269. 
39. Ribrag V, Caballero D, Ferme C, et al. Multicenter Phase II study of plitidepsin in patients with relapsed/refractory non-Hodgkin's lymphoma. Haematologica. 2013;98:357-363.

40. Eisen T, Thomas J, Miller WH Jr, et al. Phase II study of biweekly plitidepsin as second-line therapy in patients with advanced malignant melanoma. Melanoma Res. 2009;19:185-192.

41. Schoffski P, Guillem V, Garcia M, et al. Phase II randomized study of Plitidepsin (Aplidin), alone or in association with L-carnitine, in patients with unresectable advanced renal cell carcinoma. Mar Drugs. 2009; 7:57-70.

42. Baudin E, Droz JP, Paz-Ares L, et al. Phase II study of plitidepsin 3-hour infusion every 2 weeks in patients with unresectable advanced medullary thyroid carcinoma. Am J Clin Oncol. 2010;33:83-88.

43. Peschel C, Hartmann JT, Schmittel A, et al. Phase II study of plitidepsin in pretreated patients with locally advanced or metastatic non-small cell lung cancer. Lung Cancer. 2008;60:374-380.
44. Eisen T, Thatcher N, Leyvraz S, et al. Phase II study of weekly plitidepsin as second-line therapy for small cell lung cancer. Lung Cancer. 2009; 64(1):60-65.

45. Dumez H, Gallardo E, Culine S, et al. Phase II study of biweekly plitidepsin as second-line therapy for advanced or metastatic transitional cell carcinoma of the urothelium. Mar Drugs. 2009;7:451-463.

46. Toulmonde M, Le Cesne A, Piperno-Neumann S, et al. Aplidin in patients with advanced dedifferentiated liposarcomas: a French Sarcoma Group Single-Arm Phase II study. Ann Oncol. 2015;26:1465-1470.

47. Soto-Matos A, Szyldergemajn S, Extremera S, et al. Plitidepsin has a safe cardiac profile: a comprehensive analysis. Mar Drugs. 2011;9: 1007-1023.

48. Pharmamar. Aplidin - Dexamethasone in Relapsed/Refractory Myeloma (ADMYRE). Available from: https://clinicaltrials.gov/ct2/show/ NCT01102426. NLM identifier: NCT01102426. Accessed November 1, 2016 .

\section{Publish your work in this journal}

Drug Design, Development and Therapy is an international, peerreviewed open-access journal that spans the spectrum of drug design and development through to clinical applications. Clinical outcomes, patient safety, and programs for the development and effective, safe, and sustained use of medicines are the features of the journal, which has also been accepted for indexing on PubMed Central. The manuscript management system is completely online and includes a very quick and fair peer-review system, which is all easy to use. Visit http://www.dovepress.com/testimonials.php to read real quotes from published authors.

Submit your manuscript here: http://www.dovepress.com/drug-design-development-and-therapy-journal 